\title{
Medullary thyroid carcinoma and pregnancy
}

\author{
Andreea Elena DUMITRU ${ }^{1}$, Corina GICA $^{1}$, Anca Marina CIOBANU ${ }^{1,2}$, \\ Brindusa Ana CIMPOCA-RAPTIS ${ }^{1,2}$, Nicolae GICA ${ }^{1,2}$, Gheorghe PELTECU ${ }^{1,2}$, \\ Anca Maria PANAITESCU ${ }^{1,2}$ \\ ${ }^{1}$ Filantropia Clinical Hospital, Bucharest, Romania \\ 2"Carol Davila" University of Medicine and Pharmacy, Bucharest, Romania
}

\begin{abstract}
Medullary thyroid carcinoma (MTC) is a rare neuroendocrine tumor of the parafollicular cells of the thyroid gland. The tumor occurs, either sporadically or in a hereditary form, as a component of the type 2 multiple endocrine neoplasia (MEN) syndromes, MEN2A and MEN2B, both with an autosomal dominant transmission. Due to its higher aggressiveness and urgency to treat, it poses a challenge when diagnosed during pregnancy. We performed a literature review searching medical databases as UpToDate and PubMed for relevant information about diagnosis, impact, treatment, and outcome of medullary thyroid carcinoma during pregnancy. Diagnosis of MTC is established by clinical and ultrasound evaluation, followed by a fine needle aspiration biopsy. Further analyses decide whether it is a sporadic or a hereditary disease. Surgery offers the curative solution, but careful postoperative follow-up is needed for the best outcome. The decision for surgery is taken considering the DNA mutation of RET oncogene and pregnancy status. A premature planned delivery is associated with potential complications for the newborn, but it is recommended when maternal status worsens.

Conclusions. Complete resection of the thyroid tumor and any local and regional metastases is the only curative solution. Timing of the intervention is extremely important to decrease fetal outcomes. Close follow-up during the postoperative period, by both biochemical and imaging methods, is needed.
\end{abstract}

Keywords: medullary thyroid carcinoma, calcitonin, RET oncogene, pregnancy, thyroidectomy

\section{INTRODUCTION}

The most common endocrine malignancy is the thyroid carcinoma, often detected during the childbearing age. Therefore, about $10 \%$ of thyroid carcinomas occurring during the reproductive years are diagnosed during pregnancy or in the early post-partum period [1]. Thyroid nodules in women are common, about $2 \%$ percent of women between 18 and 45 years having a thyroid nodule, mostly benign ones. The prevalence of cancer in a solitary nodule ranges from $10 \%$ to $20 \%$ [2]. Most nodules are discovered by the patient or by the physician at a routine prenatal examination. The discovery of any tumor during pregnancy causes much anxiety for both pa- tient and her physician. Any evaluation or diagnostic procedure must take into account the pregnant state of the patient and the fetal concerns. However, thyroid carcinoma in young people has, generally, a good prognosis and survival rate among women with thyroid carcinoma diagnosed during pregnancy may not differ from non-pregnant women of the same age with similar disease.

Yet, pregnancy is a special situation in the field of oncology because of the need to control at least two patients at the same time, and both vulnerable. In addition, its management is particular especially during the first trimester of pregnancy.

The main objectives in the clinical monitoring of pregnant thyroid carcinoma patients are: to reach an 
adequate balance of maternal thyroid hormones that is absolutely required by the fetal central nervous system for its normal maturation; to maintain optimal levels of maternal thyroxin in order to avoid possible recurrence or spread of disease; to perform safe follow-up visits for the mother and to plan further therapy when needed [3].

When discussing about thyroid carcinoma there are two histopathological types involved: the differentiated and the medullary variant. While the differentiated thyroid carcinoma (DTC) in young people, generally, has an excellent prognosis and disease-free survival among women diagnosed during pregnancy may not differ from non-pregnant women, the medullary thyroid carcinoma (MTC) is much more aggressive with little information about its incidence and survival rates because of the large geographic variations, mainly due to its familial pattern.

Treatment for thyroid carcinoma patients is often total thyroidectomy and thus they will require adequate supplementation of both calcium and thyroxine, but timing of the surgery and further therapy is discussed in a multidisciplinary team of endocrinologist, obstetrician, maternal medicine specialist, radiologist and pediatrician or neonatologist.

\section{ETIOLOGY OF SPORADIC AND HEREDITARY MTC}

Williams [4] discovered that medullary thyroid carcinoma (MTC) is a neuroendocrine tumor originated from the neural crest derived from parafollicular C-cells of the thyroid gland, with the characteristic of secreting calcitonin [5]. Shortly after its discovery, it was recognized that the tumor occurred either sporadically or in a hereditary form as a component of the type 2 multiple endocrine neoplasia (MEN) syndromes, MEN2A and MEN2B, and the related syndrome, familial MTC (FMTC). Compared to the DTC, it is a rare form of cancer accounting for 5-10\% of all thyroid carcinomas with $70-75 \%$ of forms occurring sporadically and the rest being inherited [6].

The sporadic variant is typically diagnosed in the fourth and sixth decades of life as a solitary thyroid nodule, usually in the upper portion of the thyroid lobes due to the localization of C-cells $[5,7]$. By the time of diagnosis, the disease has already metastasized in most of these patients, presenting clinically detectable cervical lymph node involvement, symptoms of upper aerodigestive tract compression or invasion manifested by dysphagia or hoarseness, and approximately 5 to $10 \%$ have distant metastatic disease in liver, lung, bones, and, less often, brain and skin [8,9]. Biochemical tests depict calcitonin concentrations correlated with the tumor mass (high in patients with a palpable tu- mor) and also the secretion of carcinoembryonic antigen (CEA), another tumor marker that led to anti-CEA antibodies for immunotherapy.

The inherited variant is subdivided into two groups: the multiple endocrine neoplasia type $2 \mathrm{~A}$ and type $2 \mathrm{~B}$ associated with MTC, with an autosomal dominant transmission. They result from different mutations in the RET proto-oncogene. The RET proto-oncogene, located on chromosome 10q11.2, encodes a single-pass transmembrane receptor of the tyrosine kinase family and it is expressed in cells derived from the neural crest, the branchial arches, and the urogenital system [10]. Moreover, RET oncogene is associated with lung adenocarcinoma and chronic myelomonocytic leukemia, but also with Hirschsprung's disease [11]. MEN2A is associated with MTC, pheochromocytoma, and primary parathyroid hyperplasia and the penetrance of MTC is nearly 100 percent, with inter- and intrafamily variability in the specific pattern of the other disease manifestations. MEN2B shares the inherited predisposition to MTC and pheochromocytoma presented in classical MEN2A, but does not include hyperparathyroidism, with MTC occurring in almost all patients. The tumor develops at an earlier age and may be more aggressive than in MEN2A. Patients typically present with a marfanoid habitus (without having Marfan syndrome), mucosal neuromas, and intestinal ganglioneuromatosis.

\section{THYROID FUNCTION DURING PREGNANCY}

There are various changes occuring during pregnancy, but the normal course involves an increase in the renal iodine excretion, in the thyroxine binding proteins and also an augmentation of the thyroid hormone production due to stimulatory effects of hCG on the thyroid gland.

Therefore, the thyroid function tests of a healthy pregnant woman differ from a non-pregnant one. Circulating thyroxine binding globulin (TBG) and total T4 (TT4) concentrations increase by week 7 of gestation and reach a peak by approximately week 16 of gestation, remaining high until delivery [12]. During the first trimester, the maternal hCG stimulates the TSH receptor, increasing the hormone production and resulting in a subsequent reduction in serum TSH concentration. Thus, lower levels of the serum TSH is observed in as many as $15 \%$ of healthy women during the first trimester of pregnancy, reaching a decrease of about 0.1-0.2 $\mathrm{mU} / \mathrm{l}$ of the lower limit and one of about 0.5-1.0 mU// of the upper limit compared to the normal range $[13,14]$. For multiple pregancies, due to the higher concentrations of the hCG, the decrease can be even lower. 


\section{DIAGNOSIS}

Ultrasound (US) evaluation of the neck is the most important preoperative imaging study in patients with thyroid carcinoma. If any suspicion of MTC is raised, the physician should advice the patient to have the calcitonin (Ctn) and CEA levels dosed.

Fine-needle aspiration (FNA) biopsy of thyroid nodules is a useful and safe tool in the diagnosis of thyroid pathology of a pregnant patient. It is safe, inexpensive and helps distinguish those patients who can be observed safely during pregnancy from those who need surgery. FNA should be performed on thyroid nodules that are greater than $1 \mathrm{~cm}$ in size, depending on the ultrasound characteristics. The results of a fine-needle aspiration may be malignant, suspicious, benign, or indeterminate. Hamburger [15] recommends strict criteria in the interpretation: at least six clusters of benign thyroid cells on two smears from separate aspirates from different parts of the nodule to exclude malignancy reliably. Biopsy specimens from lesions greater than $4 \mathrm{~cm}$ in size can be obtained with a cutting needle, allowing more accurate histologic analysis [16]. A recommendation from Mazzaferri was to proceed with FNA in all thyroid nodules discovered prior to 20 weeks of gestation and perform this procedure after 20 weeks only if nodules grow in size during suppressive therapy. Surgery for indeterminate findings on FNA can be delayed until after delivery unless the nodule is increasing in size [17].

After having the ultrasound confirmation of a newly diagnosed thyroid nodule and the histological proof of MTC, the patient should have a direct DNA analysis of blood cells to detect the presence of a RET mutation. In case of hereditary MTC, pheochromocytoma and hyperparathyroidism should be excluded in order to establish the right treatment. Additional imaging techniques like computed tomography (CT) or contrast enhanced magnetic resonance imaging (MRI) are indicated if metastatic MTC is suspected preoperatively but cannot be performed safety prior to delivery.

\section{PRECONCEPTION AND PRENATAL COUNSELING}

Thyroid carcinoma diagnosed during pregnancy present unique challenges and requires a careful balance between making a definitive diagnosis and instituting treatment while avoiding interventions that may adversely impact the mother, the health of the fetus, or the maintenance of the pregnancy.

Preconceptional counseling and prenatal testing are available to individuals with hereditary MTC. Genetic testing can be offered to first-degree relatives of a patient with proven germline RET mutation, to parents whose infants or young children have the clinical characteristics of MEN type 2B (MEN2B), to patients with cutaneous lichen amyloidosis (CLA) and to families whose infants or young children have Hirschsprung disease (HD) [5]. While a patient may not wish to proceed with prenatal pre-implantation diagnosis, the clinician has both a legal and ethical duty to warn of genetic harms and to inform patients of all available prenatal medically appropriate options. Parents who do not wish to have prenatal RET mutation testing should be offered genetic counseling and genetic testing of their child, the timing to be determined by the treating physician in consultation with the child's parents.

\section{TREATMENT}

Patients with MTC can be cured only by complete resection of the thyroid tumor and any local and regional metastases. The issue in treating MTC during pregnancy is the timing of surgery, taking into consideration the maternal and neonatal outcome. Knowing the aggressiveness of MTC, there is a tendency to manage the malignancy more urgently than other differentiated thyroid tumors. However, a planned premature delivery may predispose the child to multiple morbidities related to prematurity.

There is no recommendation from the American Thyroid Association guidelines for the optimal timing of surgery [5]. Thus, surgery is strongly considered, as a delay in the treatment process can adversely affect outcome. Treatment options are decided considering the pregnancy's trimester and the disease aggressiveness by the specific DNA mutation in the RET proto-oncogene and the serum calcitonin levels. Pregnancy on its own can promote rapid growth of thyroid carcinoma, certain tumors having an aggressive behavior especially during first trimester, which may force the medical team to intervene and perform surgery during second trimester. For patients diagnosed during the third trimester, surgery could be safely delayed, avoiding the expected complications, as premature labor and hypotension caused by compression of gravid uterus on great vessels.

MTC can extend locally into lymph nodes and into the surrounding muscles and trachea and may invade lymphatic and blood vessels. It can metastasize in lungs, bones, and liver.

Preoperative evaluation includes thyroid and neck ultrasound to determine the extent of surgery, serum calcium to exclude hyperparathyroidism (requires concomitant surgery) and plasma fractioned metanephrines to exclude pheochromocytoma (require surgery first).

Sporadic MTC tumors are often unilateral with a single localization, while familial forms are mostly bilater- 
al and often multifocal or diffuse. For these reasons, total thyroidectomy appears to be the appropriate approach to MTC. Central neck and upper mediastinum clearance and unilateral or bilateral node dissection (depending on the extent of nodal involvement) is also advisable. Even with extensive lymph node dissection, recurrences are still common. Biochemical recurrence still can occur even when patient was operated at an early stage. Thus, close follow-up is recommended.

The role of radiotherapy is controversial as MTC is generally not believed to be radiosensitive. Radioactive iodine is ineffective because $I^{131}$ uptake by MTC cells is negligible. No reliably effective chemotherapeutic regimen has been identified. New drugs with anti-angiogenetic, anti-tyrosine kinase and anti-VEGF effects are currently under observation [1].

Postoperative management includes monitoring for possible development of hypoparathyroidism or lesions to the recurrent or superior laryngeal nerves, thyroid hormone therapy to restore/maintain euthyroidism, monitoring of serum calcitonin and carcinoembryonic antigen, periodical physical exams and neck ultrasound and additional imaging if calcitonin level are greater than $150 \mathrm{pg} / \mathrm{ml}$ [18].
In case of persistent or recurrent locoregional MTC, management include one or more neck operations, external beam radiotherapy (EBRT) or active surveillance. Patients with progressive metastatic disease who cannot be treated by surgery or radiotherapy should be considered candidates for systemic therapy.

\section{CONCLUSIONS}

Medullary thyroid carcinoma, although rare, is a severe malignancy that involves a multidisciplinary approach for the benefits of both mother and fetus. Earlier diagnosis by ultrasound, biochemical markers and fine-needle aspiration biopsy may offer better outcome.

The only option for cure is surgery and its timing is of extreme importance, depending on DNA mutation in RET proto-oncogene, calcitonin levels and gestational age. When possible, the approach of a delayed surgery for post-partum period is recommended, to avoid complications. Close surveillance and follow-up, on both mother and newborn, should be performed to assess the disease long term outcome.

Conflict of interest: none declared Financial support: none declared

\section{REFERENCES}

1. Gibelli B, Zamperini P, Proh M, Giugliano G. Management and follow-up of thyroid carcinoma in pregnant women. Acta Otorhinolaryngol Ital. 2011;31(6):358-365.

2. Norton JA, Levin B, Jensen RT. Cancer of the endocrine system. in: DeVita VT. Cancer Principles and Practice of Oncology. Philadelphia: JB Lippincott, 1993:1333.

3. Patel J, Landers K, Li H, et al. Thyroid hormones and fetal neurological development. J Endocrinol. 2011;209:1-8.

4. Williams ED. Histogenesis of medullary carcinoma of the thyroid. J Clin Pathol. 1966;19(2):114-118.

5. Wells SA Jr, Asa SL, Dralle H, et al. Revised American Thyroid Association guidelines for the management of medullary thyroid carcinoma. Thyroid. 2015;25(6):567-610.

6. Tuttle M. Medullary thyroid carcinoma: Clinical manifestations, diagnosis, and staging Available at: https://www.uptodate. com/contents/medullary-thyroid-cancerclinical-manifestations-diagnosis-and-staging

7. Gagel RF, Hoff AO, Cote GJ. Medullary thyroid carcinoma. In: Werner \& Ingbar's The Thyroid, 9th, Braverman LE, Utiger RD
(Eds). Philadelphia: Lippincott Williams \& Wilkins, 2005:967.

8. Pacini F, Castagna MG, Cipri C, Schlumberger M. Medullary thyroid carcinoma. Clin Oncol (R Coll Radiol). 2010;22:475.

9. Machens A, Hauptmann S, Dralle H. Increased risk of lymph node metastasis in multifocal hereditary and sporadic medullary thyroid carcinoma. World J Surg. 2007;31:1960.

10. Zordan P, Tavella S, Brizzolara A, Biticchi R, Ceccherini I, Garofalo S, Ravazzolo R, Bocciardi R. The immediate upstream sequence of the mouse Ret gene controls tissue-specific expression in transgenic mice. Int J Mol Med. 2006 Oct;18(4):601-8.

11. Kohno T, Ichikawa H, Totoki Y, Yasuda K, Hiramoto M, et al. KIF5B-RET fusions in lung adenocarcinoma. Nat Med. 2012 Feb 12;18(3):375-7.

12. Sahay RK, Nagesh VS. Hypothyroidism in pregnancy. Indian J Endocrinol Metab. 2012;16(3):364-370.

13. Soldin OP, Tractenberg RE, Hollowell JG, Jonklaas J, Janicic N, Soldin SJ. Trimester- specific changes in maternal thyroid hormone, thyrotropin, and thyroglobulin concentrations during gestation: trends and associations across trimesters in iodine sufficiency. Thyroid. 2004;14(12):1084-1090.

14. Kahric-Janicic N, Soldin SJ, Soldin OP, West $\mathrm{T}$, Gu J, Jonklaas J. Tandem mass spectrometry improves the accuracy of free thyroxine measurements during pregnancy. Thyroid. 2007 Apr;17(4):303-11.

15. Hamburger JI. Thyroid nodules in pregnancy. Thyroid. 1992 Summer;2(2):165-8.

16. Morris PC. Thyroid cancer complicating pregnancy. Obstet Gynecol Clin North Am. 1998 Jun;25(2):401-5.

17. Mazzaferri EL. Management of a solitary thyroid nodule. N Engl J Med. 1993 Feb 25;328(8):553-9.

18. Scollo C, Baudin E, Travagli JP, et al. Rationale for central and bilateral lymph node dissection in sporadic and hereditary medullary thyroid carcinoma. J Clin Endocrinol Metab. 2003;88:2070. 